\section{Con relación al artículo: "Lupus eritematoso sistémico con anticuerpos antinucleares negativos y anemia hemolítica". Acta Med Colomb 2010; 35: 179-182.}

\author{
Respetado Editor:
}

Leímos con mucho interés el reciente artículo publicado en la sección de "Presentación de Casos" de su revista Acta Médica Colombiana titulado "Lupus Eritematoso Sistémico con anticuerpos antinucleares negativos y anemia hemolítica" (1). El caso descrito es muy interesante y refleja la complejidad de nuestra práctica diaria y las frecuentes dificultades en el ejercicio de la medicina interna y la reumatología.

Respecto al caso reportado puede tratarse obviamente de un paciente con Lupus Eritematoso Sistémico (LES), sin embargo un análisis adicional de la información presentada permite considerar otras opciones diagnósticas. De entrada llama la atención la falta de criterios para clasificarlo como LES, cumpliendo sólo con los criterios hematológico e inmunológico. Aunque un paciente puede ser diagnosticado con LES en ausencia de criterios completos de clasificación, la falta de al menos cuatro criterios debe hacer cuestionar el diagnóstico y buscar explicaciones alternativas, máxime en ausencia de anticuerpos antinucleares (ANA).

Un diagnóstico alternativo para el cuadro clínico presentado es un síndrome antifosfolípido primario (SAF primario). La paciente tenía antecedente de una pérdida gestacional mayor de 10 semanas y anticuerpos anticardiolipinas en altos títulos además de una serología para sífilis falsamente positiva. En pacientes con SAF primario puede ocurrir anemia hemolítica autoinmune (AHAI) tal como ha sido reportado en grandes series: $5 \%$ en una serie latinoamericana (2) y $4.5 \%$ en la cohorte "Euro-phospholipid" (3). En otra serie europea de 308 pacientes con SAF primario se reportó AHAI en el $10.2 \%$ de los casos (4). En estas series también fue frecuente el reporte de anticuerpos antiRo positivos desde un $3 \%$ en europeos hasta un $18 \%$ en latinoamericanos. El consumo del complemento también es otro hallazgo reportado en SAF primario, e incluso ha sido implicado en la patogénesis de esta enfermedad. En un estudio se encontró hipocomplementemia en el $47 \%$ de 70 pacientes con SAF primario, en los cuales fue más frecuente el hallazgo de AHAI y de anticardiolipinas IgM (5).

Aunque los ANA negativo no descartan la posibilidad de LES actual o durante la evolución, pensamos que un síndrome antifosfolípido primario (o no relacionado con otra enfermedad autoinmune) explica por completo las manifestaciones clínicas y de laboratorio de la paciente reportada.

Cordialmente,

\section{Dr. Mauricio Restrepo Escobar Especialista en Medicina Interna y Reumatología Docente Auxiliar Sección de Reumatología Departamento de Medicina Interna Facultad de Medicina, Universidad de Antioquia Medellín, Colombia.}

\section{Referencias}

1. Builes CE, Durango IC, Velásquez CJ. Lupus eritematoso sistémico con anticuerpos antinucleares negativos y anemia hemolítica. Acta Med Colomb 2010; 35: 179-82.

2. Mejía-Romero R, García-Carrasco $\mathbf{M}$, Galarza-Maldonado $\mathbf{C}$, Santos $\mathbf{P}$ Mendoza-Pinto C, Escárcega RO, et al. Primary antiphospholipid syndrome in Latin American mestizo patients: clinical and immunologic characteristics and comparison with European patients. Clin Rheumatol 2008; 27: 891-7.

3. Cervera R, Piette JC, Font J, Khamashta MA, Shoenfeld Y, Camps MT, et al. Antiphospholipid syndrome. Clinical and Immunologic Manifestations and Patterns of Disease Expression in a Cohort of 1,000 Patients. Arthritis Rheum 2002; 46: 1019-27.

4. Rottem M, Krause I, Fraser A, Stojanovich L, Rovensky J, Shoenfeld Y. Autoimmune hemolytic anaemia in the antiphospholipid syndrome. Lupus 2006; 15: $473-7$.

5. Ramos-Casals M, Campoamor MT, Chamorro A, Salvador G, Segura S, Botero JC, et al. Hypocomplementemia in systemic lupus erythematosus and primary antiphospholipid syndrome: prevalence and clinical significance in 667 patients. Lupus ; 13: 777-83. 


\section{Respuesta del autor}

Respetado Editor:

En primer término queremos agradecer el gran aporte académico hecho a nuestro reporte de caso por el doctor Mauricio Restrepo Escobar; sea esta la oportunidad para resaltar las cualidades profesionales del doctor Restrepo, a quien tengo el gusto de conocer personalmente y es, sin duda, una figura promisoria de la reumatología colombiana.

En resumen, el doctor Restrepo tiene toda la razón; revisando la literatura, es cierto que el síndrome antifosfolípido puede explicar las manifestaciones clínicas (anemia hemolítica) y paraclínicas (anticuerpos anti-Ro e hipocomplementemia) de la paciente reportada. Me permito agregar dos referencias recientes al respecto:

Oku y colaboradores (1) estudiaron 36 pacientes con SAF primario y los compararon con 42 controles y encontraron niveles de complemento sérico más bajos en estos pacientes: C3: 81.07 (17.86) vs 109.80 (22.76) mg/dL, p<0.001; C4: 13.04 (8.49) vs 21.70 (6.96) mg/dL, p<0 .001; $\mathrm{CH}(50): 31.32$ (8.76) vs $41.40(7.70) \mathrm{U} / \mathrm{mL}, \mathrm{p}<0.001$.

Comellas-Kirkerup L y colaboradores (2) analizaron 55 pacientes con SAF primario y encontraron $25 \%$ de individuos con anemia hemolítica.

Para confirmar este diagnóstico, deben repetirse los anticuerpos anticardiolipinas y realizar los anticuerpos anti- $\beta 2$ glicoproteína I. También deben repetirse el perfil inmunológico y definir si hay cambios en el seguimiento clínico de la paciente que permitan que cumpla los criterios clasificatorios de Lupus Eritematoso Sistémico, que creemos sigue siendo un diagnóstico diferencial válido, como se argumentó en la discusión del reporte de caso (3).

Cordialmente,

Dr. Carlos Jaime Velásquez Franco Especialista en Medicina Interna y Reumatología Universidad de Antioquia. Docente de Medicina Interna Facultad de Medicina, Escuela de Ciencias de la Salud, Universidad Pontificia Bolivariana. Reumatólogo Clínica Universitaria Bolivariana y Hospital Pablo Tobón Uribe. Medellín, Colombia.

\section{Referencias}

1. Oku K, Atsumi T, Bohgaki M, Amengual O, Kataoka H, Horita T, et al. Complement activation in patients with primary antiphospholipid syndrome. Ann Rheum Dis 2009; 68: 1030-5.

2. Comellas-Kirkerup L, Hernández-Molina G, Cabral AR. Antiphospholipidassociated thromb. ocytopenia or autoimmune hemolytic anemia in patients with or without definite primary antiphospholipid syndrome according to the Sapporo revised classification criteria: a 6-year follow-up study. Blood 2010; 116: 3058-63.

3. Builes CE, Durango IC, Velásquez CJ. Lupus eritematoso sistémico con anticuerpos antinucleares negativos y anemia hemolítica. Acta Med Colomb 2010; 35: $179-82$. 